\title{
Small HER2 Positive Breast Cancer: When is Enough?
}

Small node negative HER2 Positive tumors are defined as T1 $(<2 \mathrm{~cm})$ and N0 (node negative). The above cohort is increasing with more awareness and acceptance of mammography screening. This group in itself is a heterogeneous population such as $\mathrm{T} 1 \mathrm{a} / \mathrm{b}$ versus $\mathrm{T} 1 \mathrm{c}$, Grade I versus II/III and estrogen receptor (ER) positive versus negative tumours. The role of adjuvant therapy for these women is a long-standing dilemma for clinicians due to the lack of prospective randomized trials and a poor representation of this group in pivotal trials. Even guidelines are inconsistent on chemotherapy regimen and the duration of trastuzumab for this subset.

There is increasing evidence from several retrospective studies for an inferior outcome in these patients with recurrence rates as high as $15 \%-30 \%$ after $5-10$ years. ${ }^{[1]}$ In a British Columbia data set ${ }^{[2]}$ of N0 breast cancers, positive HER2 status was an independent predictor of breast cancer death in 10 years in a multivariable model, with odds ratio $(\mathrm{OR})$ of $2.03(P=0.003)$ and in a European Institute of Oncology ${ }^{[3]}$ population of pT1abN0 breast cancers, it was an independent predictor of 5 years disease-free survival (DFS) with OR of $2.5 \quad(95 \%$ confidence interval $[\mathrm{CI}]: 0.9-6.5 ; P=0.09)$. The available evidence for the efficacy of trastuzumab for these patients has limitations such as subgroup analysis of large randomized trials, retrospective nature, small numbers, few events in trials, and differing end points or durations of follow up. Five large randomized Phase III multicenter studies have shown that the addition of trastuzumab to chemotherapy results in decreased recurrence and better overall survival (OS). The proportion of TIN0 tumors and their survival outcome (subgroup analyses) compared to the overall group is depicted in Table 1.

\section{Deescalation of Chemotherapy}

The APT trial ${ }^{[9]}$ was the first prospective investigation of a reduced intensity adjuvant treatment regimen (weekly paclitaxel and trastuzumab [TH] for 12 weeks, followed by completion of 1 year of trastuzumab $[\mathrm{H}]$ ) in small, node-negative HER2+ disease following standard breast surgery. Majority of the patients in this trial were T1N0 with $8.9 \%$ of these had T size $2-3 \mathrm{~cm}$. Survival free from invasive disease, the primary end point of the trial, was 98.7\% (95\% CI: $97.7 \%-99.8 \%)$ at 3 years, supporting $\mathrm{TH}$ as a highly effective regimen in this patient population. Another prospective, single-arm Phase II trial ${ }^{[10]}$ has looked at a more intensive regimen in early stage HER2+ breast cancer. In this trial, patients were treated in the adjuvant setting with four cycles of docetaxel/cyclophosphamide/ trastuzumab, followed by every 3 week trastuzumab to complete a year of therapy. The primary endpoint was DFS at 2 years, but follow-up was long enough to calculate 3-year survival estimates. Overall 3-year DFS and OS were $96.9 \%$ and $98.7 \%$, respectively. Among node-negative patients with primary tumor size less than or equal to $1 \mathrm{~cm}$, 3-year DFS and OS were both 100\%.

\section{Deescalation of Trastuzumab Duration}

Adjuvant trastuzumab is approved across the globe for 1 year duration as standard treatment. There have been attempts at reducing this duration [Table 2]. The arguments for a shorter duration of exposure may be supported by

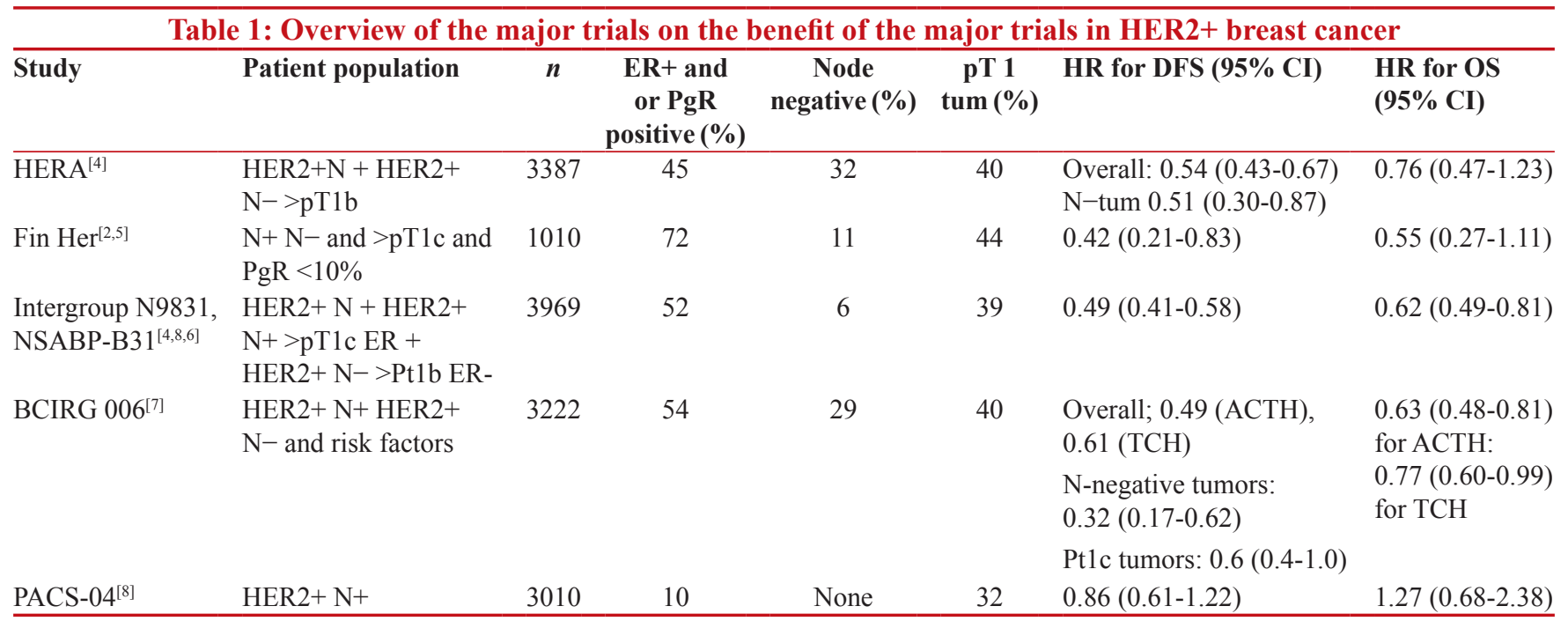

HR given for the additional benefit for adjuvant trastuzumab compared with no such treatment. HR - Hazard ratio; CI - Confidence interval; DFS - Disease-free survival; OS - Overall survival; ER - Estrogen receptor; ACTH - Docetaxel and trastuzumab 


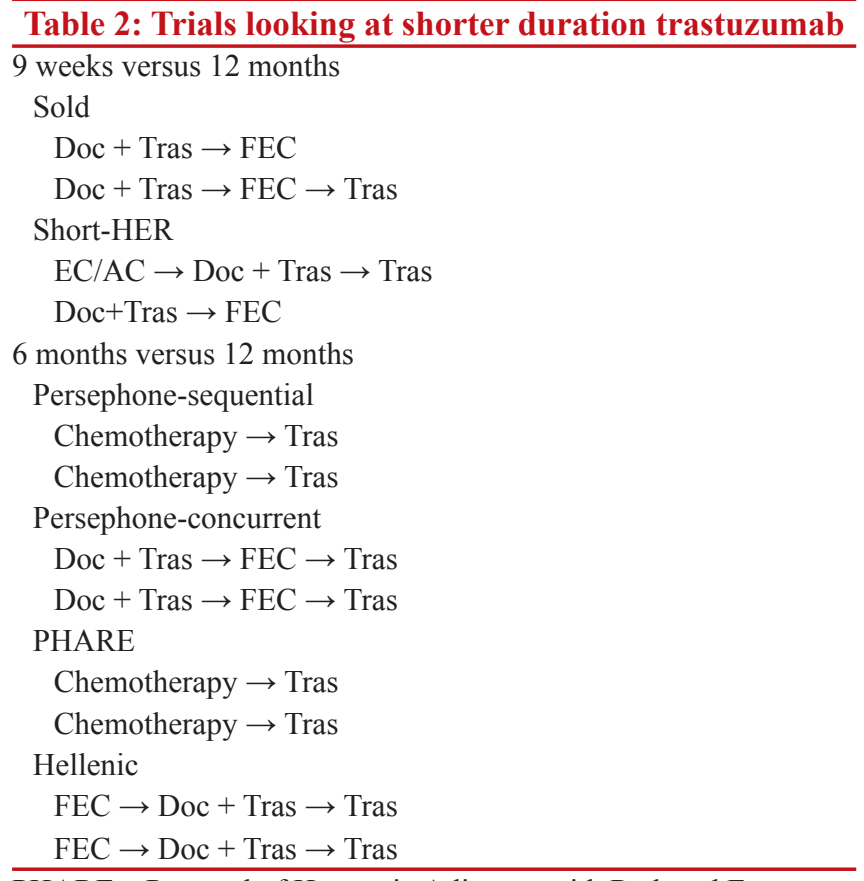

PHARE - Protocol of Herceptin Adjuvant with Reduced Exposure

cardiac safety and cost effectiveness and especially relevant for this subset of patients.

FINHER approach reported by Joensuu et al.$^{[5]}$ showed that a brief course of trastuzumab administered concomitantly with docetaxel is safe and effective, but the study cohort included patients who were either node positive or had $\mathrm{T}$ size $>2 \mathrm{~cm}$ high-risk node negative tumors. The Protocol of Herceptin Adjuvant with Reduced Exposure trial ${ }^{[1]}$ evaluated the efficacy of 6 versus 12 months of trastuzumab treatment. In this noninferiority trial, 3400 patients who had started trastuzumab were randomly assigned at 6 months to complete the standard 12 months of therapy or to stop therapy at the 6-month time point. The 95\% CI hazard ratio (HR) for noninferiority was set at 1.15 . However, 6 months of trastuzumab did not meet this threshold, with an HR of $1.28(P=0.029)$. Of note, although statistical noninferiority of 6-month duration was not established, the absolute benefit of longer duration in DFS and OS was small. Subsequently, a subgroup analysis of this study was published. In this analysis, there was a suggestion that in patients with very low risk ( $\mathrm{T}<2 \mathrm{~cm}$ and N0) and low-risk ( $\mathrm{T} \leq 2 \mathrm{~cm}$ or $1-3$ nodes) 6 months and 12 months of trastuzumab did not result in markedly different outcomes. $^{[12]}$ Hellenic Oncology Research Group conducted a randomized study to compare the efficacy of 12 versus 6 months of adjuvant trastuzumab administered concurrently with dose-dense chemotherapy in women with node-positive or high-risk node-negative early breast cancer. Although this trial also failed to demonstrate the noninferiority of 6 versus 12 months administration, it had many limitations such as small sample size and a relatively high noninferiority margin of $8 \%{ }^{\left[{ }^{[13]}\right.}$ Results of other trials of shorter trastuzumab duration are forthcoming.

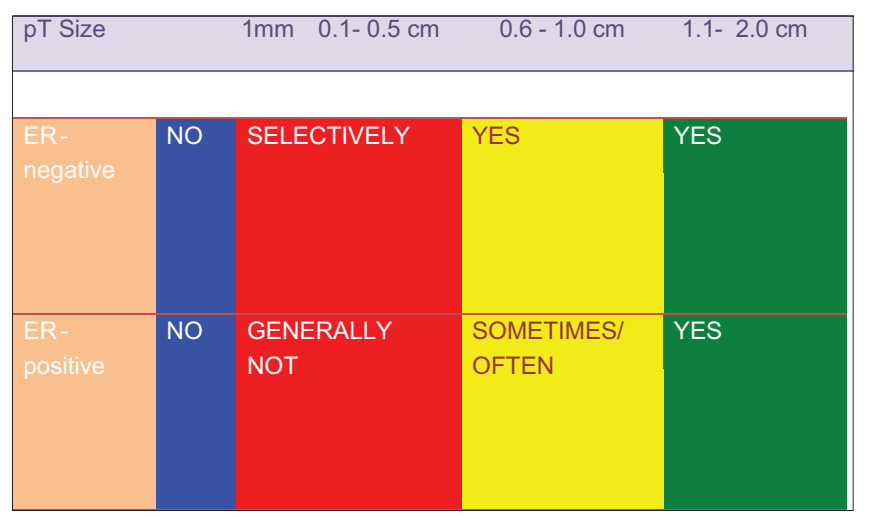

Figure 1: Proposed schema for adjuvant therapy of Stage 1 HER2+ breast cancer

The proposed schema for Adjuvant therapy of Stage 1 HER2+ breast cancer is shown in Figure 1.

In summary, both retrospective and meta-analysis data suggest a benefit to trastuzumab treatment in small and/or node-negative HER2+ tumors. The questions of "how low and how much" and tumor size thresholds remain open to debate. Available data also suggests that, although durations shorter than 1 year are not clearly noninferior to 1 year of adjuvant trastuzumab, the absolute benefits of longer duration are small and may not exist in small, node negative tumors. Thus, durations shorter than 1 year may be appropriate in resource constrained settings. The potential role of novel HER2 directed therapies in this cohort is not clear. Greater integration of translation research and gene signatures may provide further insight for this subset in the future.

\section{Randeep Singh, Sudeep Gupta ${ }^{1}$}

Department of Medical Oncology, Artemis Cancer Centre, Gurugram, ${ }^{\prime}$ Department of Medical Oncology, Tata Memorial Hospital, Mumbai, Maharashtra, India E-mail: sudeepgupta04@yahoo.com

\section{References}

1. Joerger M, Thürlimann B, Huober J. Small HER2-positive, node-negative breast cancer: Who should receive systemic adjuvant treatment? Ann Oncol 2011;22:17-23.

2. Chia S, Norris B, Speers C, Cheang M, Gilks B, Gown AM, et al. Human epidermal growth factor receptor 2 overexpression as a prognostic factor in a large tissue microarray series of node-negative breast cancers. J Clin Oncol 2008;26:5697-704.

3. Curigliano G, Viale G, Bagnardi V, Fumagalli L, Locatelli M, Rotmensz N, et al. Clinical relevance of HER2 overexpression/amplification in patients with small tumor size and node-negative breast cancer. J Clin Oncol 2009;27:5693-9.

4. Untch M, Gelber RD, Jackisch C, Procter M, Baselga J, Bell R, et al. Estimating the magnitude of trastuzumab effects within patient subgroups in the HERA trial. Ann Oncol 2008;19:1090-6.

5. Joensuu $\mathrm{H}$, Kellokumpu-Lehtinen PL, Bono P, Alanko T, Kataja V, Asola R, et al. Adjuvant docetaxel or vinorelbine with or without trastuzumab for breast cancer. N Engl J Med 2006;354:809-20.

6. Perez EA, Romond EH, Suman VJ, Jeong JH, Sledge G, 
Geyer CE Jr., et al. Trastuzumab plus adjuvant chemotherapy for human epidermal growth factor receptor 2-positive breast cancer: Planned joint analysis of overall survival from NSABP B-31 and NCCTG N9831. J Clin Oncol 2014;32:3744-52.

7. Slamon D, Eiermann W, Robert N. Phase III randomized trial comparing doxorubicin and cyclophosphamide followed by docetaxel (ACT) with doxorubicin and cyclophophamide followed by docetaxel and trastuzumab (ACTH) with docetaxel, carboplatin and trastuzumab (TCH) in women with Her2neu positive early breast cancer patients: BCIRG 006 study. Cancer Res 2009;69(24 Suppl):Abstract62.

8. Spielmann M, Roché H, Delozier T, Canon JL, Romieu G, Bourgeois $\mathrm{H}$, et al. Trastuzumab for patients with axillary-node-positive breast cancer: Results of the FNCLCC-PACS 04 trial. J Clin Oncol 2009;27:6129-34.

9. Tolaney SM, Barry WT, Dang CT, Yardley DA, Moy B, Marcom PK, et al. Adjuvant paclitaxel and trastuzumab for node-negative, HER2-positive breast cancer. N Engl J Med 2015;372:134-41.

10. Jones SE, Collea R, Paul D, Sedlacek S, Favret AM, Gore I Jr., et al. Adjuvant docetaxel and cyclophosphamide plus trastuzumab in patients with HER2-amplified early stage breast cancer: A single-group, open-label, phase 2 study. Lancet Oncol 2013;14:1121-8.

11. Pivot X, Romieu G, Debled M, Pierga JY, Kerbrat P, Bachelot T, et al. 6 months versus 12 months of adjuvant trastuzumab for patients with HER2-positive early breast cancer (PHARE): A randomised phase 3 trial. Lancet Oncol 2013;14:741-8.
12. Kramar A, Bachelot T, Madrange N, Pierga JY, Kerbrat P, Espié $\mathrm{M}$, et al. Trastuzumab duration effects within patient prognostic subgroups in the PHARE trial. Ann Oncol 2014;25:1563-70.

13. Mavroudis D, Saloustros E, Malamos N, Kakolyris S, Boukovinas I, Papakotoulas P, et al. Six versus 12 months of adjuvant trastuzumab in combination with dose-dense chemotherapy for women with HER2-positive breast cancer: A multicenter randomized study by the Hellenic Oncology Research Group (HORG). Ann Oncol 2015;26:1333-40.

This is an open access article distributed under the terms of the Creative Commons Attribution-NonCommercial-ShareAlike 3.0 License, which allows others to remix, tweak and build upon the work non-commercially, as long as the author is credited and the new creations are licensed under the identical terms.

\begin{tabular}{|l|l|}
\hline \multicolumn{2}{|c|}{ Access this article online } \\
\hline Quick Response Code: & Website: \\
& www.ijmpo.org \\
\cline { 2 - 2 } & DOI: \\
\hline
\end{tabular}

How to cite this article: Singh R, Gupta S. Small HER2 positive breast cancer: When is enough?. Indian J Med Paediatr Oncol 2018;39:1-3. 